\title{
Practical experience from a regional secure unit
}

\author{
Adrian James, Senior Registrar in Forensic Psychiatry, Fromeside Clinic, \\ Blackberry Hill, Stapleton, Bristol BS16 1ED
}

The Royal College of Psychiatrists' Working Party on Medical Audit has emphasised the multidisciplinary nature of patient care in psychiatry (Royal College of Psychiatrists, 1989). Much has been published on individual audit topics and on definitions of the various aspects of audit such as peer review, performance indicators and quality assurance. The essential element of audit is its systematic nature and little has been written concerning an overall structure for clinical audit within an individual psychiatric setting. It is not sufficient to start with 'a simple project with minimum of cost in terms of both time and money expended', as recommended by the Royal College of Psychiatrists' Working Party. What is needed is an overall structure for regular review so that the systematic nature of the process is not lost and that recommendations from audit meetings are reviewed regularly for their usefulness and level of implementation.

This paper presents an overall structure for clinical audit within a regional secure unit. This process is up and running at Fromeside Clinic, a regional secure unit based in Bristol and covering the northern part of the South Western Regional Health Authority. This is a 30-bedded unit with two full-time consultant forensic psychiatrists.

\section{Aims}

(a) To improve our service to patients and other 'customers'.

(b) To generate an open atmosphere for argument and debate concerning procedures on the unit.

(c) To educate each member of the multidisciplinary team in the working practices of other disciplines and facilitate co-operation.

\section{Essential elements}

(a) Clinical audit involves members of all disciplines working within a regional secure unit.

(b) An atmosphere must be created whereby a frank exchange of ideas and criticisms is possible without threatening the integrity of individuals. Service deficiencies must be identified which require attention rather than individuals who feel responsible for a shortfall in service provision. (c) The first step in audit is to look at procedures and set a timetable for review. The reasons for certain activities proceeding in a particular way must be investigated before embarking on innovative minimum standards programmes. Audit is an evolving 'hands on' process and too much forward planning is counter productive.

(d) A small multidisciplinary team of people is appointed to take overall responsibility for the control of the process. Their first responsibility is to set up an audit programme, identify topics for regular review and set a timetable covering the first year of an audit programme.

(e) Small multidisciplinary teams are appointed to lead a discussion on specific audit topics. Each group should have an identified leader who is responsible for 'delivering the goods'. These groups are responsible for collecting and analysing data on a particular topic, highlighting strengths and weaknesses, and proposing mechanisms for change and the setting of minimum standards.

(f) Monthly meetings are set at times convenient for all members of the staff team. Having collected the data on individual topics, a representative of each individual audit group presents any findings and recommendations for overall comment and review by the clinic staff as a whole. Review is conducted on a multidisciplinary basis because all aspects of our individual working practices affect others working in different disciplines.

(g) Review of technical aspects of the working practices of a member of a particular discipline may be difficult within a multidisciplinary setting and will require audit by professionals of the same discipline on a district wide basis. An example would be aspects of the working practices of our one physiotherapist which are not amenable to satisfactory review by other members of the multidisciplinary team.

\section{Audit topics}

(a) Medical note-keeping and procedures for medical practice. This includes the setting of a minimum standard of medical note-keeping.

(b) Nursing procedures and note-keeping. 
(c) Multidisciplinary practice and note-keeping including the level of attendance of patients at activities within the Clinic, including assessment of patient satisfaction.

(d) Special incident audit. This includes the numbers of violent incidents, seclusion, absconding and use of 'prn' medication. Groups investigating these topics will be expected to formulate ways of collecting data, presenting data, comparing rates of occurrence of certain incidents over time, and with other units, and recommending changes in clinical practice.

(e) Statistical audit. This is the analysis of routinely collected health service data. It includes the total number of referrals to the service, the source of referral, speed of response, responsible health district, admission numbers, bed occupancy, demographic data, length of admission and eventual disposal. The use of a data base is essential.

\section{Further procedural details}

A timetable is set for monthly meetings. The first could be audit of nursing and multidisciplinary notes and procedures, the second medical procedures and minimum standards for note-keeping, the third special incident audit, and the fourth concerning statistical audit, and so on. This process repeats itself with the agenda for review of a particular topic being set four months in advance by the previous meeting.

The review at this clinic is essentially on an intraclinic basis. We have two consultants and two wards and they can audit each other's practice. Minutes are recorded with anonymity preserved for both patients and staff. These are followed up with regular brief reports to the district audit committee. Along with this we have three monthly meetings with our sister secure unit, the Butler Clinic, which provides a service for the southern part of the South Western Region. Audit topics are agreed in advance and our practice compared and procedures reviewed. The need to compare our practice with units outside our regional health authority is essential and will be planned in due course. There will no doubt be a need for independent review on topics we are struggling with in accordance with the recent Department of Health circular (DOH, 1991). Finally, the contribution to the audit process by bodies such as the Mental Health Act Commission, Mental Health Review Tribunals and Health Advisory Services should be facilitated and welcomed as an adjunct to improving patient care.

\section{Acknowledgements}

I would like to thank Drs Arden Tomison and Robert Reeves for their helpful comments.

\section{References}

Department of Health (1991) Medical Audit in the Hospital and Community Health Services. Assuring the quality of medical care: Implementations of Medical and Dental Audit in the Hospital and Community Health Services Circular No. HC(91)2.

Royal College of Psychiatrists (1989) Preliminary report on medical audit. Psychiatric Bulletin, 13, 577-580.

\section{New Booklist}

The third annual supplement to the List of Books Suitable for a Psychiatric Library 1983-1987 has been prepared by the College Library. These lists are compiled for the assistance of trainees and psychiatric tutors as well as colleague librarians seeking assistance in identifying suitable books for inclusion in their libraries. It is available from the Publications Department of the College. Price $£ 2.00$. 